\section{Le tâtonnement "marshallien" dans les premiers écrits d'Oskar Lange}

Emeric Lendjel*

\section{Résumé}

Cet article revient sur le célèbre article de O. Lange [1936-37] en prenant appui sur ses premiers écrits (Lange [1932], [1935a], [1935b]) afin de montrer qu'il développe une représentation originale du processus marchand. Ces premiers articles mettent en lumière deux points méconnus de ses travaux. 1/ Tout d'abord, Lange développe un modèle original décrivant le processus d'ajustement temporel de l'offre dans les secteurs industriels (en complément du diagramme du cobweb se rapportant principalement aux secteurs agricoles). 2/ Cet article montre ensuite que Lange articule ces différents modèles dans le cadre d'une conception originale du tâtonnement. L'article montre que cette conception relève davantage de l'approche marshallienne que de l'approche walrassienne habituelle.

\section{Abstract}

Based on Lange's neglected first writings (Lange [1932], [1935a], [1935b]), this article shows that Lange developed an original conception of market adjustment processes in his famous 1936-7 article. In particular, two points are showed here. 1/ Lange developed an original model that described the lagged and progressive adjustment process of supply in industrial sectors (completing the cobweb diagram that concern mainly agricultural sectors). $2 /$ Lange tried to articulate these different an particular models in a general frame which corresponds to the tâtonnement. The article shows that this conception is related to a marshallian approach rather than to a walrassian one.

\section{Classification JEL : B 220, B 240, C 620, D 490, D 590}

La contribution d'Oskar Lange au débat sur la rationalité du calcul économique en régime socialiste dans les années trente est, en général, assez bien connue ${ }^{1}$. Pourtant, à notre connaissance, personne n'a cherché à situer le fameux article de 1936-7, "On the Economic Theory of Socialism", par rapport à ses travaux antérieurs. Certes, Lange réagit, à travers cet article, aux critiques "théoriques" et

\footnotetext{
* Adresse électronique : lendjel@yahoo.com. Je tiens à remercier M. DeVroey et P. Bridel ainsi que les deux rapporteurs pour leurs critiques, commentaires et suggestions. Cet article n'engage, comme à l'accoutumée, que son auteur.

1 Voir Bergson [1948] et [1967], Brus et Kowalik [1970], Roberts [1971], Elliott [1976], Rothbard [1976], Vaughn [1980], Cave et Hare [1981], Murrell [1983], Kowalik [1987a] [1987b], Lavoie [1985], Temkin [1989], Donzelli [1993], Palermo [1998].
}

"pratiques" qu'adressent Von Mises, Hayek et Robbins à la rationalité d'une allocation non-marchande des ressources dans une économie socialiste. Mais cette réaction n'est pas seulement circonstancielle.

En effet, elle s'enracine profondément dans la démarche intellectuelle de Lange telle qu'on peut la percevoir au travers ses premiers articles. On peut en effet distinguer deux volets dans cette démarche. Le premier concerne la formation des grandeurs économiques. Lange développe dès 1928 une réflexion personnelle sur cette question qui l'amènera à étudier de près le tâtonnement ${ }^{2}$. Le second volet se rapporte à l'organisation d'une économie socialiste. En 1934, il propose, en collaboration avec Marek Breit, une représentation d'une organisation possible de l'économie socialiste. En 1935, il se donne les moyens d'utiliser les catégories néoclassiques pour étudier théoriquement cette organisation. Par conséquent, l'article de 1936-1937 n'est pas seulement un article de circonstance, destiné à prendre à contre-pied la critique des libéraux.

Davantage, cet article gagne une nouvelle profondeur théorique lorsqu'on le replace dans la dynamique des contributions précédentes de Lange. En général, cet article est perçu comme s'insérant dans la tradition walrassienne de l'équilibre général (Vaughn [1980], p. 535, Temkin [1989], p. 201). Dans cette perspective, Murell reproche à la solution de Lange de s'inscrire dans un cadre statique (Murell [1983] p. 97). La clause de suspension des échanges avant l'équilibre (Cave et Hare [1981], p. 101) ou l'existence d'une institution centrale analogue à la figure du commissaire priseur (Vaughn [1980], p. 541, Temkin [1989], p. 201) sont autant de caractéristiques qui inscrivent, pour ces auteurs, la solution de Lange dans le cadre de la théorie walrassienne de l'équilibre général. Or, à suivre cette lecture walrassienne du texte de 1936-7, on se heurte à une ambivalence qui semble entacher la procédure par "essai et erreur" décrite par Lange, ambivalence qui se cristallise autour de la question de l'erreur. Le tâtonnement oscille en effet incessamment entre une procédure atemporelle, sans échanges ni production effectifs en dehors de l'équilibre, où l'erreur ne peut donc qu'être fictive, et une procédure temporelle, permettant des "essais et des erreurs" qui n'ont rien de fictifs. Pourtant, si l'on rapproche l'article de 1936-7 des articles précédents de Lange, une autre lecture devient possible.

L'élément important qui caractérise ces premiers articles est leur caractère syncrétique. En effet, les théories de Marx côtoient celles de Marshall, de Walras ou encore celles de l'école autrichienne. Ce syncrétisme conduit Lange à introduire un questionnement marshallien dans le cadre de l'équilibre général $^{3}$. Dès lors, l'hypothèse que nous avançons est la suivante : Lange proposerait une lecture marshallienne du tâtonnement, lui permettant d'aborder d'une manière originale le problème de la stabilité de l'équilibre général.

2 Rappelons que, depuis Walras, peu d'auteurs ont abordé le tâtonnement. Chez Pareto et Barone, le tâtonnement est assimilé à une procédure que l'on peut utiliser dans des systèmes économiques différents comme la "concurrence" ou le "régime collectiviste". Cette interprétation participe d'un questionnement naissant à l'époque sur les procédures d'allocation des ressources en économie centralement planifiée. Taylor [1929], la principale source de Lange [1936-7], s'inspire directement de la réflexion de Barone sur le tâtonnement [1908]. Voir Lendjel [1998], chap. II et [1999].

${ }^{3}$ Selon Kowalik, Lange était tenté par la réalisation d'une "grande synthèse" (Kowalik [1987a], p. 128). 
Nous empruntons à De Vroey les caractéristiques d'une approche "marshallienne" de l'équilibre général. Schématiquement, cet auteur retient quatre traits distinctifs de cette approche. 1/ Contrairement à l'approche walrassienne où l'économie se définit comme un "marché unique géant",

"l' «économie marshallienne» [est] définie comme un entrelacement de marchés séparés, chacun associé à un bien particulier et à un dispositif spécifique pour la formation de l'équilibre du marché. La monnaie est ce qui les lie ensemble" (De Vroey [1999a], p. 416) ${ }^{4}$.

2/ L'économie marshallienne se distingue de l'approche walrassienne par le rôle qu'elle confère au temps. Le temps n'est plus simplement un temps logique, mais un temps "réel", caractérisé par l'irréversibilité des actes (De Vroey [1999b], p. 175). 3/ L'approche marshallienne hiérarchise son analyse de l'économie :

"[1]e cachet de l'approche marshallienne du fonctionnement de l'économie est qu'elle procède en deux étapes. Le fonctionnement des marchés particuliers est analysé dans la première étape, alors que la coordination ou l'assemblage des différents résultats partiels est assignée à la seconde étape de la recherche" (De Vroey [1999a], p. 417).

4/ L'approche marshallienne distingue deux concepts d'équilibre : l'équilibre de court terme ou du "jour de marché" et l'équilibre de long terme ou "équilibre normal". Ces deux types d'équilibre résultent de deux processus d'ajustement distincts. Le premier a cours sur le marché du jour et se caractérise par la verticalité de la courbe d'offre. Ce processus d'ajustement de la demande à l'offre conduit à l'équilibre de marché. Le second s'effectue sur le "long terme" (à partir de deux jours) et se caractérise par des échanges en déséquilibre, à faux prix. Au terme de ce second processus d'ajustement de l'offre et de la demande, le prix atteint sa "valeur normale" (ibid.). 5/ Enfin, une cinquième caractéristique peut être ajoutée dans laquelle s'enracine toute la tradition de l'économie industrielle et une partie d'approche dite des organisations. I s'agit d'une part, de l'étude de l'industrie en tant qu'organisation meso-économique et, d'autre part, de l'organisation interne de la firme, ce que Marshall désignait par "business organisation" (Ménard [1990]) En ce sens, l'approche marshallienne mobilise dans l'analyse économique les caractéristiques organisationnelles des firmes et industries.

Nous verrons que la réflexion de Lange sur la formation des grandeurs économiques s'inspire, pour une grande part, de cette approche marshallienne. Un certain nombre d'hypothèses utilisées par Marshall pour garantir la convergence du processus marchand vers l'équilibre, comme celle de la constance de l'utilité marginale de la monnaie, ou celles de "constance des données" et de "no-carry-over", sont en effet reprises par Lange. L'emploi de ces hypothèse dans une perspective marshallienne permet de lever l'ambivalence - évoquée plus haut - qui marque le statut de l'erreur dans la "procédure par essai et erreur".

Davantage, notre hypothèse permet de souligner l'évolution des caractéristiques que l'on a associé au concept de tâtonnement. Désormais associé à la formulation canonique de Samuelson [1941], le tâtonnement n'a pas toujours eu le sens qu'on lui donne de nos jours. Ainsi, Walras ne développe pas la même conception du tâtonnement que Barone, Pareto, Lange ou même Samuelson. Les contours et les

\footnotetext{
${ }^{4}$ Toutes les traductions sont de notre fait, sauf mention contraire dans la bibliographie.
}

caractéristiques du tâtonnement se sont modifiés au cours de l'histoire (Lendjel [1998]). En lui donnant sa configuration canonique, Samuelson a en quelque sorte "stabilisé" l'oscillation conceptuelle qui caractérisait le problème de la dynamique et du processus marchand (Weintraub [1991]). Nous chercherons ainsi à montrer que la définition que retient Lange du tâtonnement n'est pas tant "walrassienne", comme on pourrait le croire au regard de l'utilisation du terme proprement dit, mais s'inspire davantage de Marshall. Le concept de tâtonnement renvoie ainsi, pour Lange, à l'idée d'un cadre général permettant d'articuler des processus marchands différents selon les secteurs : diagramme du Cobweb pour les secteurs agricoles et diagramme "marshallien" - selon le terme de Lange - pour les secteurs industriels. Les secteurs sont en effet caractérisés par des temporalités, des institutions, des caractéristiques organisationnelles et comportementales différentes qui doivent être pris en compte lors de l'examen du processus d'ajustement marchand de l'ensemble de l'économie. Le tâtonnement de Lange ne correspond donc pas à la conception que nous en avons aujourd'hui 5

Pour le montrer, il nous faut revenir sur ces premiers articles afin de reconstituer ces premiers jalons importants de la démarche intellectuelle de Lange. Nous évoquerons donc brièvement, dans un premier temps, le syncrétisme qui caractérise ces premiers développements (1. Le syncrétisme de Lange). Nous examinerons ensuite, dans un deuxième temps, les premières représentations que propose Lange de la formation des grandeurs économiques en soulignant leur influence marshallienne (2. Les premières représentations de la formation des grandeurs économiques sous influence marshallienne). Nous montrerons enfin, dans un troisième temps, que Lange développe une lecture marshallienne du tâtonnement dans "On the Economic Theory of Socialism" (3. Une lecture marshallienne du tâtonnement)

\section{Le syncrétisme de Lange}

La démarche intellectuelle de Lange frappe par son caractère syncrétique, au moins dans ses premiers écrits. L'auteur la justifie dans deux de ses premiers articles : "L'interdépendance générale des prévisions en économie et la méthode d'isolement", paru en 1932 et "L'économie Marxienne et la Théorie Economique Moderne" publié en $1935^{6}$. Dans le premier article, Lange analyse les avantages et les inconvénients des deux approches dominantes de l'équilibre: l'équilibre partiel et l'équilibre général. Son syncrétisme transparaît dans son objectif même : insérer l'approche de Marshall au sein du cadre de la théorie de l'équilibre général ${ }^{7}$. Dans le deuxième article, il transparaît dans sa volonté de justifier

5 Dans cette perspective, la réflexion qu'ouvre Lange sur la question de la stabilité de l'équilibre général constitue une des bifurcations possibles qu'aurait pu prendre la théorie économique, bifurcation que ne mentionne pas Weintraub dans son ouvrage Stabilizing Dynamics (1991).

6 Littéralement, "Die allgemeine Interdependenz der Wirtschaftsprognosen und die Isolierungsmethode" (Lange [1932]) et "Marxian Economics and Modern Economic Theory" (Lange [1935b]).

7 Pour situer l'entreprise de Lange, il faut évoquer ici les deux principaux reproches adressés aux théories de l'équilibre dans la période de l'entre-deux guerres : leur caractère "statique" et leur "irréalisme" (Gislain et Steiner [1995], p. 38) 
l'utilisation des catégories néoclassiques pour l'étude de l'économie socialiste. Un certain nombre d'éléments émerge ainsi de ces deux textes et témoigne d'une démarche syncrétique.

\subsection{La "méthode d'isolement" dans le cadre de la théorie de l'équilibre général}

Le syncrétisme de Lange se caractérise d'abord par la volonté explicite de prendre acte de l'existence de plusieurs courants théoriques en économie et de n'en retenir que certains aspects. Dans cette perspective, le mérite de la théorie de l'équilibre général est le suivant :

"Walras et Pareto ont, les premiers, montré les conséquences méthodologiques du fait de l'interdépendance générale de tous les biens économiques et engendré un appareil méthodique grâce à l'appréhension de l'interdépendance de tous les biens économiques par un système d'équations simultanées"(Lange [1932], p. 52).

En d'autres termes, le "méthodisme" de l'école de Lausanne tient à l'utilisation des mathématiques pour pouvoir traiter de l'interdépendance des phénomènes économiques, affirme Lange en se référant à Pareto. C'est d'ailleurs ce qui la distingue des théories classiques, y compris Marxienne (ibid.). Cela étant, la théorie de l'équilibre général souffre d'un grave défaut, aux yeux de Lange : sa "stérilité" pour analyser des problèmes concrets (ibid., p. 56). En effet,

"l'analyse concrète d'un problème particulier ne peut pas être conduite sans la méthode d'isolement" (ibid.).

A l'inverse de la théorie de l'équilibre général, affirme Lange, la "méthode d'isolement" de Marshall, qui ne se caractérise pas principalement par le recours aux mathématiques, permet précisément l'étude des phénomènes particuliers ${ }^{8}$. Cette méthode consiste à faire abstraction des interdépendances afin d'etudier, par l'analyse, un phénomène particulier (ibid., p. 53). Mais, c'est là en même temps son principal inconvénient :

"[1]a faute de la méthode d'isolement réside dans le fait qu'elle se représente les grandeurs économiques particulières à travers une ou quelques grandeurs économiques déterminées, pendant que toutes les grandeurs économiques restantes sont considérées par la pensée comme constantes par le moyen de la clause «ceteris paribus»" (ibid., p. 54).

8 Marshall résumait ainsi non sans humour le rôle qu'il attribuait aux mathématiques en économie : "1) Usez-en comme d'une sténographie plutôt que comme d'un instrument de recherche; 2) Tenez-vous-y jusqu'à ce que vous ayez abouti à un résultat; 3) Traduisez le en langage courant ; 4) Illustrez les résultats par des exemples importants dans la vie réelle; 5) Brûler les calculs; 6) Si vous ne réussissez pas à réaliser le $4^{\circ}$, brûler les résultats du $3^{\circ}$; c'est ce que j'ai souvent fait moi-même." (Lettre à Bowley du 27 fev. 1906, cité par Granger [1955], 294).
Dès lors, comment lever la clause ceteris paribus une fois le phénomène analysé ? Ainsi, après avoir montré les intérêts et les limites des deux approches, Lange propose de les hiérarchiser en abordant l'équilibre général à partir de l'étude préalable des marchés particuliers. Cette approche, marquée par le "cachet" de l'approche marshallienne (De Vroey [1999a], p. 417), suppose cependant de rendre légitime l'isolement d'un marché particulier.

En évoquant les tentatives de Marshall et d'autres auteurs pour fonder la méthode partielle, Lange relève que leur problème est de trouver un critère mesurable permettant de savoir à partir de quel seuil un marché peut être considéré comme isolé. . Schématiquement, son propos consiste alors à dire que l'interdépendance entre les marchés peut exister à des degrés divers. Pour résoudre des problèmes pratiques, il faut pouvoir caractériser ces degrés de dépendances entre des secteurs précis. Lange propose donc de retenir un critère théorique et statistique pour répondre à cette question ${ }^{10}$. L'interdépendance se mesure par l'élasticité croisée de deux biens, la variation de la quantité consommée d'un bien sur un marché entrânant une variation sur celle d'un autre marché (Lange [1932], p. 64). Lorsque cette élasticité est nulle, le marché du bien $\mathrm{X}$ est indépendant du marché du bien $\mathrm{Y}$, et peut donc être analysée isolément. Lorsque cette élasticité s'approche de un en valeur absolue, les deux biens sont interdépendants (ibid., p. 65). Les deux marchés doivent donc s'analyser ensemble. Ce critère théorique s'accompagne d'une procédure de test statistique (voire économétrique) de manière à caractériser les relations entre les biens. Ce faisant, Lange estime disposer d'un critère permettant d'appliquer ou non la méthode d'isolement.

Ainsi, le syncrétisme de Lange s'exprime ici à travers la possibilité d'utiliser une méthode dans un cas et une autre dans le cas contraire.

\subsection{Le caractère opératoire des théories de l'équilibre et la dimension institutionnelle de la théorie "Marxienne"}

Le syncrétisme de Lange se retrouve dans l'article de 1935 dans lequel il s'agit de rendre légitime l'emploi des théories "bourgeoises" de l'équilibre pour analyser une économie socialiste. Il répond en cela aux attaques de Shibata [1933] qui conteste la pertinence des concepts des théories de l'équilibre pour décrire ce type d'économie ${ }^{11}$. Ici, le syncrétisme confronte les théories de l'équilibre d'une part et la théorie "Marxienne" d'autre part. Trois points caractérisent cette approche en 1935.

- Comme en 1932, la première caractéristique de l'approche syncrétique de Lange en 1935 est de prendre acte de l'existence de plusieurs courants théoriques en économie. Ce dernier pense en effet que l'économie est constituée de théories différentes, dont les objets, les méthodes et les concepts diffèrent

${ }^{9}$ Remarquons que Lange ne mentionne pas le problème de la reconstruction d'un équilibre général à partir d'équilibres partiels.

10 Friedman reviendra sur ce problème dans son célèbre article "The Marshallian Demand Curve" [1949]. Je dois ce rapprochement à M. De Vroey.

11 Pour une discussion de la référence à Marx des modèles d'économie socialiste, voir Elliott [1976]. 
d'une théorie à l'autre. Loin de vouloir les hiérarchiser entre elles, Lange considère que les théories peuvent coexister dans la diversité de leur méthode et de leurs concepts à partir du moment où leur objet est différent ${ }^{12}$. Si "la théorie de l'équilibre" se donne pour objet l'étude de l'allocation des ressources rares à usages alternatifs (Lange [1935b], p. 192), la théorie de Marx traite davantage de l'évolution des systèmes économiques (ibid., p. 193). La différence d'objet provient en fait d'une approche différente du rôle du temps historique dans l'analyse. La théorie de l'équilibre raisonne dans un cadre "statique", à partir de "données" constantes.

"La théorie économique telle que l'ont développé les écoles autrichiennes, marshalliennes et celle de Lausanne est essentiellement une théorie statique de l'équilibre économique, analysant le processus économique dans un système de données constantes et le mécanisme par lequel les prix et les quantités produites s'ajustent d'elles-mêmes au changement de ces données. Les données elles-mêmes, qui sont psychologiques (les échelles de préférence des consommateurs), techniques (les fonctions de production), et institutionnelles (les formes et la distribution de la propriété des facteurs de production, le système monétaire et bancaire, etc.) sont considérées comme extérieures au champ de l'économie théorique" (ibid., p. 192).

La théorie marxienne, au contraire, cherche à connaître l'évolution historique de ces données. Elle cherche à "fournir en plus une théorie de l'évolution économique" (ibid., p. 193) ${ }^{13}$. Elle ne se contente pas de repérer statistiquement cette évolution, mais de lui en fournir une explication théorique.

"Ainsi, l'anticipation de la course future des événements déduite par la théorie Marxienne n'est pas une extrapolation mécanique d'une tendance purement empirique, mais une anticipation basée sur la reconnaissance d'une loi de développement et est, avec quelques réserves, non moins rigoureuse qu'une anticipation basée sur la théorie statique de l'équilibre économique telle que, par exemple, l'anticipation qu'une augmentation d'un prix conduit, sous certaines circonstances, à une baisse du montant d'une marchandise demandée" (ibid.).

La théorie marxienne et la théorie de l'équilibre sont donc aussi rigoureuses l'une que l'autre, mais concernent des objets différents. Elles sont donc compatibles entre elles, puisque leur champ d'analyse ou leur objet est différent.

- Comme en 1932, la deuxième caractéristique syncrétique de la démarche de Lange consiste à vouloir introduire de l' "institutionnel" dans la théorie "formelle" de l'équilibre. Encore faut-il préciser ce qu'entend Lange par "formel", en 1935. Traitant d'objets distincts, la théorie de l'équilibre - général - et celle de Marx produisent pourtant des énoncés qui sont tous deux "formels". Ces "principes formels", abstraits, sont susceptibles de s'appliquer à n'importe quel type d'économie.

12 Voir sur ce point Elliott [1976], p. 60.

13 Durant son séjour à Harvard, Lange fut sous le tutorat de Schumpeter (Kowalik [1987a], p. 126).
"Les principes formels de la théorie de l'équilibre économique sont les mêmes pour n'importe quel type d'économie d'échange. Le système d'équations walrassiennes est indifféremment applicable à une économie capitaliste ou a une économie marchande simple ["einfache Warenproduktion"]. Le fait que les personnes qui possèdent les services productifs du travail et ceux du capital (la force de travail et les moyens de production dans la terminologie Marxienne) soient les mêmes ou non affecte, bien entendu, les résultats concrets du processus d'équilibre économique, mais non ses aspects théoriques formels. Mais c'est également vrai de la formulation de la théorie de l'équilibre économique dont se servait Marx, i. e. de la théorie de la valeur travail. Cette théorie aussi s'applique indifféremment à n'importe quel type d'économie d'échange, pourvu qu'elle soit parfaitement concurrentielle" (Lange [1935b], p. 197).

Ainsi, les équations de la théorie de l'équilibre général se situent au même niveau d'abstraction que certains éléments de la théorie marxienne. Le caractère formel d'un énoncé ne dépend donc pas de l'utilisation des mathématiques. Un énoncé peut être formel sans être mathématique. On ne peut donc pas critiquer la théorie de l'équilibre général en raison de sa dimension formelle, comme le fait Shibata ([1933], p. 107).

En revanche, on peut lui reprocher sa faiblesse sur la prise en compte des composantes institutionnelles, contrairement à la théorie marxienne. En ce sens, le "formel" s'oppose à l' "institutionnel" ou au "concret" pour Lange. On retrouve ici le principal reproche qu'il adressait à la théorie de l'équilibre général en 1932. Alors que le formel renvoie aux caractéristiques abstraites et universelles de systèmes économiques, l'institutionnel ou le concret se rapporte aux caractéristiques particulières à chaque système. Le principal défaut des théories de l'équilibre provient donc de l'absence de spécification institutionnelle des systèmes.

"Tant que la théorie de l'équilibre économique est simplement une théorie de la distribution de ressources rares entre des usages alternatifs, elle n'a besoin d'aucune donnée institutionnelle, puisque les considérations pertinentes peuvent être déduites de l'exemple de Robinson Crusoé. Jusqu'ici l'économie n'est même pas une science sociale. Quand la théorie économique traite du processus de détermination des prix la spécification des données institutionnelles est très générale. [...] Mais les conséquences de l'ajout de données institutionnelles supplémentaires, distinguant le capitalisme des autres formes de l'économie d'échange - par exemple l'existence d'une classe de gens ne possédant aucun moyens de production -, sont rarement examinées" (Lange [1935b], p. 192).

Lange s'inscrit donc ici nettement du côté de Marx - et implicitement de Marshall - lorsqu'il insiste sur la nécessité de prendre en compte les "données institutionnelles" dans l'analyse. Résoudre des problèmes concrets dans une économie socialiste suppose en effet d'incorporer ce type de données dans l'analyse. On retrouvera cette caractéristique en 1936-7. 
La dernière caractéristique de l'approche syncrétique de Lange réside dans sa volonté de fonder des concepts opératoires. Au fond, peu importe la marque d'origine d'une théorie du moment qu'elle permet de résoudre des problèmes concrets ${ }^{14}$. Si l'article est construit sur une apparente symétrie ${ }^{15}$ et se conclut sur les principaux résultats de la théorie marxienne, il sert en fait à justifier l'utilisation future des concepts de la théorie de l'équilibre pour résoudre les problèmes de l'économie socialiste. En effet, pour tout problème de gestion concrète des ressources disponibles, Lange soutient, contre Shibata ([1933], p. 107), que la théorie de l'équilibre - principalement dans sa version marshallienne - est plus opératoire que la théorie marxienne. La raison vient essentiellement de son objet. Puisque l'objet de la théorie de l'équilibre n'est autre que l'allocation des ressources rares à des fins alternatives, cette théorie est plus appropriée pour résoudre les problèmes d'organisation des systèmes socialistes.

"Il est évident que l'économie marshallienne offre davantage pour l'administration courante du système économique de la Russie Soviétique que l'économie Marxienne, bien que cette dernière soit certainement la meilleure base pour anticiper l'avenir du Capitalisme. Ainsi, l'économie théorique moderne, en dépit de son indiscutable origine "bourgeoise", a une signification universelle" (Lange [1935b], p. 191).

Ainsi, le syncrétisme de Lange s'avère au fond justifié par une sorte de pragmatisme. La mobilisation de telle ou telle théorie dépendra du cas concret à résoudre ${ }^{16}$.

L'utilisation syncrétique des catégories issues de cadres théoriques différents constitue donc une caractéristique de la démarche de Lange. Grâce à cette démarche, nous allons voir que Lange donne, en 1936-7, un contenu original à une approche marshallienne du tâtonnement en équilibre général.

\section{Les premières représentations de la formation des grandeurs économiques sous influence marshallienne}

La réflexion de Lange qui le conduira à appliquer le tâtonnement à une économie socialiste s'enracine dans ses premiers écrits. Sa thèse (1928) en porte déjà la marque, puisqu'elle porte sur les cycles des affaires en Pologne entre 1925 et 1927. Puis, en s'intéressant à cette question, Lange lit - et citera - les travaux de Ricci [1930], Schultz [1930] et Tinbergen [1930] sur le diagramme du cobweb et ceux de Rosenstein-Rodan [1929] sur le rôle des délais d'ajustements dans la formation des grandeurs économiques. Ces travaux sont en effet reliés à la question des cycles dans les secteurs agricoles ${ }^{17}$.

\footnotetext{
14 C'est dans ce pragmatisme que s'enracine probablement la démarche syncrétique de Lange.

15 L'article de [1935b] suit en effet la structure suivante : 1. Les échecs des deux théories, 2. Les objets distincts des deux théories, 3. La dimension formelle des deux théories.

16 En cela, le syncrétisme de Lange n'est pas sans évoquer la méthodologie de Marshall.

17 Voir Lendjel [1998], chapitre III.
}

Lange publie ensuite en 1935 un article intitulé "Formes de l'ajustement de l'offre et équilibre économique" ${ }^{18}$, qui traite de l'impact sur l'équilibre économique et sa stabilité des différents modes d'ajustement de l'offre. La question que soulève Lange dans cet article provient directement de ce qu'implique le diagramme du cobweb pour la stabilité de l'équilibre général. Lange relève que le diagramme du cobweb implique la possibilité d'un processus marchand ne convergeant pas vers l'équilibre. En effet, Ricci - tout comme Tinbergen - le montre explicitement dès 1930 dans une série de graphiques (Ricci [1930], 656), la convergence ou non du processus marchand, sur un marché agricole donné caractérisé par un ajustement séquentiel de l'offre, dépend de la pente des deux courbes d'offre et de demande ${ }^{19}$. Si un marché peut "pratiquement" ne pas parvenir à l'équilibre même si cet équilibre existe "théoriquement", qu'en est-il pour l'ensemble des marchés, au niveau de l'équilibre général (Lange [1935a], p. 359)?

Pour s'attaquer à ce problème, Lange adopte ici implicitement le cadre d'analyse qu'il se donnait en 1932. Comme nous l'avons vu, il soulignait dans ce texte que, si la méthode d'équilibre général est logiquement la plus solide, elle s'avère "stérile" pour "résoudre les problèmes pratiques" (Lange [1932], p. 56). En effet, chaque secteur économique est doté de caractéristiques qui lui sont propres - notamment temporelles - et que l'on ne peut forcément transposer à un autre. Plaquer un cadre général sur des processus marchands particuliers ne peut donc être fructueux. Lange choisit ainsi délibérément d'adopter la "méthode d'isolement" pour étudier les modes pratiques de formation des grandeurs d'équilibre dans les différents secteurs économiques. Grâce à cette méthode, Lange espère ensuite parvenir à une représentation pertinente de l'interdépendance générale à partir de l'analyse des processus marchands qui caractérisent les différents secteurs.

\subsection{Une représentation "marshallienne" du processus marchand dans les secteurs industriels}

Le diagramme du cobweb comporte un inconvénient majeur, pour Lange : il ne concerne pour ainsi dire que les seuls secteurs agricoles (Lange [1935a], p. 360) ${ }^{20}$. En effet, le diagramme du cobweb ne décrit que des secteurs caractérisés par un ajustement discontinu, séquentiel, de l'offre, comme l'industrie agricole dont la production est scandée par le rythme discontinu des récoltes. On le sait, un déplacement de la courbe de demande - perturbant le point d'équilibre initial - induit un processus d'ajustement de l'offre qui n'est pas instantané. L'offre s'adapte en effet avec un temps de retard (une certaine période doit s'écouler avant que l'on puisse récolter ce que l'on a semé). Ce délai, ajouté au fait que le producteur

18 Littéralement, "Formen der Angebotsanpassung und wirtschaftliches Gleichgewicht" (Lange [1935a]).

${ }^{19}$ En fait, Lange ne cite que Leontief ([1934], p. 305) dans cet article et non pas Ricci ou Tinbergen (Lange [1935a], p. 358). Les diagrammes du cobweb étant largement connus, il est inutile d'y revenir ici.

20 En fait, le sens qu'attribue Lange au terme "secteur" renvoie plutôt aujourd'hui au concept de "branche" employé dans la comptabilité nationale, i.e. un regroupement d'activités homogènes produisant le même bien. 
programme son offre en fonction du niveau des prix de la période précédente, induit un processus d'ajustement de l'offre à la demande qui n'est pas forcément convergent.

Dans les secteurs industriels, en revanche, l'ajustement de la production se fait d'une manière plus adaptative, en répondant progressivement aux modifications de la demande. Lange tente alors de développer un modèle caractérisé par une adaptation progressive de l'offre aux conditions de la demande. Cette représentation "marshallienne", selon le terme de Lange, repose sur l'idée que la réaction de l'offre à une variation du prix nécessite des délais pour que la production atteigne la quantité requise. Le graphique suivant fournit (Fig. 4) une représentation décrivant l'ajustement progressif de l'offre à un déplacement de la courbe de demande et, ainsi, du prix courant vers le prix d'équilibre.

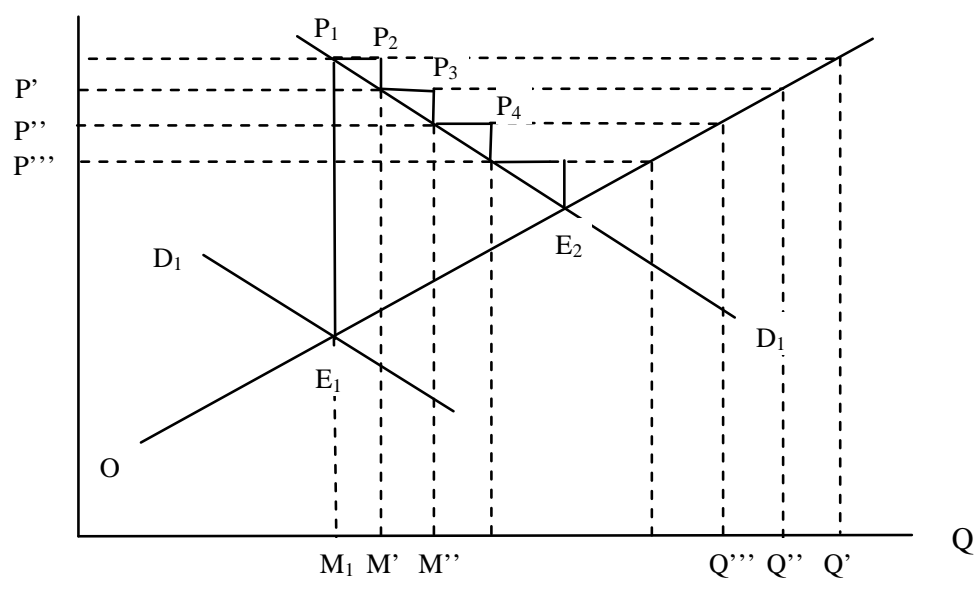

Fig. 4

Le point de départ est le même que pour le diagramme du cobweb : un déplacement soudain de la courbe de demande, nécessitant une réaction de l'offre. Or, Lange suppose que cet ajustement, qu'il soit continu ou même séquentiel, nécessite un délai. La mise en œuvre d'une production supplémentaire ne peut se faire du jour au lendemain, du fait de capacités limitées de production et de l'importance des coûts fixes (Lange [1935a], p. 361). Elle suppose l'acquisition de machines, de matières premières, l'embauche d'une maind'œuvre supplémentaire, etc. afin d'adapter l'offre à la demande. Lange attribue implicitement une technique productive donnée aux entreprises, de manière à justifier la constance de la courbe d'offre tout au long du processus d'ajustement. Ces délais d'adaptation se traduisent par l'inertie de la production sur le court terme ${ }^{21}$. En raison de cette inertie, le processus d'ajustement décrit une courbe en escalier.

${ }^{21}$ Lange ([1935a], p. 361) fait référence à Marshall lorsqu'il cherche à établir l'importance des coûts fixes dans la fonction de production relativement à la période d'ajustement considérée. Il distingue ainsi la courbe d'offre de court terme de celle du long terme. Plus la période d'ajustement du marché est longue, moins l'entreprise est contrainte dans le choix de ses combinaisons productives. Cela se traduit, au niveau
Suite à l'augmentation brutale de la demande, les entreprises ne peuvent réagir, dans un premier temps, que par l'augmentation du prix de marché. "Puisque le bien $\mathrm{OM}_{1}$ ne peut s'accrôtre immédiatement, le prix de marché augmente jusqu'à $\mathrm{M}_{1} \mathrm{P}_{1}$ " (ibid., p. 360). La courbe d'offre étant verticale sur le court terme, la demande détermine donc le prix qui solde le marché ${ }^{22}$. Le prix se fixe ainsi au niveau $\mathrm{P}_{1}$ le long de la courbe de demande. Mais, au regard des profits que les entrepreneurs peuvent espérer obtenir, ils se fixent l'objectif de répondre intégralement à cette nouvelle demande, en formant l'hypothèse que le prix sera le même dans la période suivante.

"Les entrepreneurs orientant leur production en fonction du prix de marché, ils souhaitent ainsi produire la quantité OQ'. Pour que l'offre puisse atteindre la quantité

OQ', on a besoin d'attendre un intervalle de temps donné, par exemple quatre mois" (ibid.).

L'objectif ne peut donc être atteint qu'au terme d'une période donnée. Entre-temps, les entreprises vont développer leur production. Cela n'est possible qu'en

"augmentant les achats de matière première et de force de travail et en augmentant l'exploitation des capacités productives de l'usine existante. Ce faisant, la production peut être augmentée de OM' pendant par exemple un mois" (ibid.).

L'augmentation de la production permet de répondre partiellement à l'augmentation de la demande. Mais, du fait de cet accroissement des quantités produites, la demande se trouve moins contrainte que le mois précédent. Le prix, tout en restant favorable aux entreprises, tombe au niveau $\mathrm{P}_{2}$ ( $\left.\mathrm{M}^{\prime} \mathrm{P}^{\prime}\right)$. Puisque les profits sont toujours positifs, les entreprises vont poursuivre l'augmentation de la production, mais en réduisant leurs objectifs.

"Par la suite, le prix de marché tombe à M'P' et les entrepreneurs ne souhaiterons produire encore que la quantité OQ". Au bout d'un mois supplémentaire, les nouvelles machines peuvent être mises en service et la production peut ainsi être augmentée jusqu'à $\mathrm{OM}$ ". Le prix tombe à $\mathrm{M} " \mathrm{P} "$ et les entrepreneurs ne souhaitent plus produire maintenant que la quantité OQ"'. [...] Ainsi se poursuit le processus d'ajustement, jusqu'à ce que finalement, par exemple au bout de quatre mois, l'ajustement soit totalement réalisé et le point d'équilibre $\mathrm{E}_{2}$ atteint" (Lange [1935a] p. 360)

Le processus d'ajustement progressif de l'offre se poursuit jusqu'à l'équilibre. Le prix baisse ainsi progressivement du fait de la lenteur de l'ajustement de l'offre. Dans la mesure où les objectifs sont san

micro-économique, par le choix de la taille optimale de la firme sur la longue période, puis au nivea méso-économique, par le nombre de firmes produisant dans le secteur considéré

22 Ce raisonnement suppose, comme le note Kaldor ([1934], p. 127), que les biens soient périssables et/ou qu'il n'y ait pas d'effets de report dans le temps. 
cesse révisés, la durée totale de l'ajustement pourrait théoriquement excéder les quatre mois initialement prévus $^{23}$. Pourtant, Lange soutient que l'équilibre est atteint au bout des quatre mois prévus.

Lange propose alors de fournir une formulation algébrique ou "mathématique" de ce processus représenté géométriquement. Ainsi,

"[1]e processus d'ajustement continu de l'offre peut être également représenté mathématiquement. Soit $p$ le prix, $x$ la quantité produite, $t$ le délai d'ajustement écoulé. Davantage, soit $t_{0}$ la période d'ajustement complète, c'est-à-dire celle nécessaire à l'établissement de la combinaison optimale de tous les facteurs de productions, et soit $x_{0}$ la quantité déterminée à travers la courbe d'offre momentanée. Considérant $t$ comme un paramètre, nous obtenons ainsi un groupe de courbe d'offre que l'on peut représenter par l'équation suivante :

$$
p=\varphi\left(x, t, x_{0}\right) \quad[1] \text { " (ibid., p. 362). }
$$

Si l'on fait varier la période, on obtient une série de courbes d'offre, que Lange considère comme bornée d'une part par la courbe d'offre "momentanée" (verticale, en raison de l'existence de coûts fixes), et d'autre part par la courbe d'offre de long terme où tous les facteurs de production peuvent varier de manière à obtenir la combinaison productive optimale.

"Pour $t=0$, l'équation (1) donne une courbe d'offre momentanée, pour $t \geq t_{0}$, une courbe d'offre de long terme. Pour $0<t<t_{0}$, nous obtenons le groupe des courbes de court terme" (ibid.)

Si la fonction de demande se définit simplement par $p=f(x)$ [2] et que le prix proposé par l'offre doit être égal au prix de demande, le processus de formation des grandeurs d'équilibre peut alors être représenté par l'équation suivante (ibid., p. 363) :

$$
f(x)=\varphi\left(x, t, x_{0}\right) \quad[3]
$$

En faisant varier $t$ de zéro jusqu'à $t_{0}$, Lange propose une représentation mathématique du processus de formation des grandeurs d'équilibre décrit par le secteur considéré.

"Pour chaque valeur de $t$ on obtient une valeur déterminée de $x$ et, à travers l'image de cette valeur par la fonction de demande, une valeur déterminée pour $p$ " (ibid.).

Son raisonnement s'arrête là puisqu'il s'agit d'une simple piste de recherche qu'il poursuivra en $1944^{24}$ L'important, pour lui, consiste à montrer que l'on peut se donner une représentation mathématique correspondant au graphique évoqué plus haut (Fig. 4).

\footnotetext{
23 On pourrait même imaginer qu'ils n'atteignent jamais le point d'équilibre, puisque les entrepreneurs révisent à chaque fois leurs objectifs à la baisse, tout comme dans le paradoxe de Zenon.

24 En 1941, Samuelson analyse la stabilité des "marchés multiples" en faisant l'hypothèse que les vitesses d'ajustement des marchés sont identiques et constantes, de manière à retrouver les conditions de stabilité de Hicks. En 1944, Lange reprend cette analyse et les formulations mathématiques de Samuelson en montrant que ces conditions sont compatibles avec des vitesses d'ajustement différentes selon les marchés. L'intérêt
}

Lange tire deux conclusions importantes de cette représentation "marshallienne" du processus d'ajustement de l'offre dans les secteurs industriels.

1/ Ce processus se caractérise par l'absence de fortes fluctuations.

"Ainsi, l'ajustement ne s'effectue pas à travers des oscillations, mais au contraire selon une courbe en escalier qui longe la partie $\mathrm{P}_{1} \mathrm{E}_{2}$ de la courbe de demande" (ibid.).

Du fait du comportement adaptatif des entrepreneurs, révisant constamment leurs objectifs en fonction de l'état de la demande, la production s'accroît progressivement pour un prix décroissant régulièrement. On pourrait même imaginer qu'en augmentant le nombre d'ajustements et en raccourcissant leur durée, on puisse atteindre, en passant à la limite, un ajustement continu du prix le long de la courbe de demande (ibid.).

2/ Cette représentation du processus d'ajustement de l'offre et du prix résout, aux yeux de Lange, le problème de la stabilité pour un secteur industriel isolé. En effet, quelle que soit la forme - et la pente des courbes d'offre et de demande, Lange pense avoir montré que le processus est toujours convergent. Cela tient pour une part à l'inertie de la production et, pour une autre part, au comportement de l'entrepreneur qui adapte sa production en fonction du prix et du profit de la période précédente.

Pourtant, l'étude de la stabilité de l'équilibre partiel suppose un examen complémentaire que Lange n'aborde pas. Comment réagit l'offre lorsqu'elle se trouve en situation excédentaire par rapport à la demande ? En fait, on peut facilement imaginer la réponse que fournirait Lange à cette question. Du fait de l'existence de capacité productive excédentaire, l'offre se retrouve avec un excès de machines, de main œuvre, de stocks d'invendu, etc. Elle est donc obligée d'accepter un niveau de prix très bas, avec des profits négatifs. Pour ajuster ses capacités productives, l'offre va tenter de diminuer ses coûts fixes, en vendant ses machines, et ses coûts variables, en baissant le niveau de la production et en écoulant les stocks. En raréfiant les quantités disponibles des biens qu'elle vend, le prix s'élèvera progressivement au fur et à mesure que les capacités productives de l'offre s'ajusteront au niveau de la demande. On retrouve ainsi le processus d'ajustement progressif de l'offre vers le point d'équilibre.

Ainsi, en développant une représentation du processus d'ajustement de l'offre dans un secteur industriel, Lange est amené à résoudre de manière originale la question de la stabilité de l'équilibre. Contrairement au diagramme du cobweb, susceptible de représenter des oscillations "explosives", la représentation "marshallienne" s'avère toujours convergente. Ce faisant, Lange nous propose une conception du processus marchand qui se situe à l'opposé de nos conceptions usuelles. La convergence de

de ce résultat s'inscrit, pour Lange, dans le débat sur les mérites respectifs du marché et du socialisme. En intégrant dans ses calculs le paramètre des vitesses d'ajustement différentes selon les secteurs, une autorité planificatrice peut assurer à l'économie un sentier de croissance optimal procurant à chaque instant le même bien-être total à la communauté qu'un système de concurrence atomistique (Lange [1944], p. 99). La continuité dans les recherches de Lange est ici patente. 
ce dernier résulte en effet non pas d'une flexibilité parfaite de l'offre mais au contraire de son inertie et de sa lenteur.

Reste à savoir comment les processus d'ajustement de l'offre dans les différents secteurs s'articulent entre eux au niveau général.

\subsection{Le tâtonnement comme cadre général pour les processus d'ajustement sectoriels}

Pour Lange, l'articulation entre les différents processus d'ajustement s'effectue au sein d'un cadre général que nous désignerons sous le terme de "tâtonnement". Pourtant, le terme proprement dit n'apparaît pas encore en 1935 et il faut attendre l'année suivante pour le voir figurer explicitement. Si, malgré tout, nous l'utiliserons, c'est essentiellement pour deux raisons. 1/ La représentation que se donne Lange du processus d'ajustement général correspond à celle qu'il donnera en 1936 en lui associant le terme de tâtonnement. 2/ En 1936, Lange se réfère explicitement à ses travaux antérieurs, notamment ceux de l'année précédente, soulignant ainsi la continuité - et la proximité temporelle - de ses investigations. Il n'y a donc pas lieu de supposer une inflexion significative de sa pensée sur ce point. L'hypothèse la plus plausible est donc qu'il se donne en 1936 un concept - le tâtonnement - pour désigner le processus général d'ajustement qu'il décrivait implicitement en 1935. Dans ce texte en effet, il ne s'agit pas, pour Lange, de rappeler les caractéristiques du tâtonnement, mais d'en analyser la convergence. La description qu'il fournit du tâtonnement en 1935 est donc particulièrement succincte et ne constitue qu'une ébauche de ce qu'il exposera en 1936-7.

Deux points préfigurent en effet la description que Lange donnera du tâtonnement en 1936.

- La première caractéristique, essentielle à ses yeux, du tâtonnement réside - déjà - dans "le caractère paramétrique des prix" (Lange [1935a], p. 359). Le prix est un paramètre en ce que nul agent ne peut individuellement le modifier, même les agents en situation de monopole. En effet, même un monopole sur un marché donné n'est pas en mesure de pouvoir exercer son influence sur l'ensemble des prix, dans tous les secteurs économiques. Par conséquent, tous les agents considèrent les prix comme des paramètres sur la base desquels ils prennent leur décision. Autrement dit, ils sont, comme pour Walras, "price-takers". Cette fonction paramétrique des prix sera reprise en 1936-7, pour souligner la nécessité de l'existence des prix en tant qu' "indice de choix alternatifs" dans une économie socialiste. La caractéristique première du processus d'ajustement du prix courant vers le prix d'équilibre est donc le caractère paramétrique de l'information principale des agents.

En simulant l'enchaînement des réactions des agents, le prix apparaît successivement comme un paramètre - sur la base duquel ils établissent leurs plans ou leurs "dispositions" - et comme le résultat produit par ces plans (ibid.). Le tâtonnement ne s'enclenche que lorsque l'équilibre économique est perturbé sur un ou plusieurs marchés. En ce cas, il se crée un "processus d'oscillations" analogue à celui décrit par le cobweb (ibid.).

"Les prix se modifient à la suite de l'écart entre offre et demande et perturbent ainsi les paramètres à partir desquels les entrepreneurs et les consommateurs ont établi leurs dispositions. Ces dispositions doivent donc être modifiées, ce qui change l'offre et la demande des différents biens. Ce faisant, les prix se modifient de nouveau, ce qui conduit à un autre changement des dispositions des entrepreneurs et consommateurs. Seulement, les prix se modifient de nouveau, et ainsi de suite. Que ces oscillations des prix et des quantités de biens convergent vers une position d'équilibre, ce n'est nullement évident a priori. Pour pouvoir en dire quelque chose, on a besoin de données supplémentaires, soit sur les élasticités d'offre et de demande, soit sur les anticipations des individus relatives aux futurs prix fixés" (ibid.).

Dans ce processus, les prix se modifient en fonction de la demande nette du bien en assumant leur fonction paramétrique, Lange ne se donnant pas la peine de préciser le sens de variation de ces prix selon le signe de la demande nette. Cette description du processus de tâtonnement s'avère, on le voit, assez succincte, mais s'achève sur une interrogation essentielle concernant la stabilité de l'équilibre général.

- $\quad$ Le second point concerne le rôle de cadre que Lange confère au tâtonnement. Ce rôle n'est pas affirmé explicitement, mais transparaît dans l'analyse qu'il développe de la convergence du tâtonnement vers l'équilibre général. Deux éléments permettent de donner un peu de chair à cette idée.

A la suite du diagramme du cobweb, Lange montre que le tâtonnement peut également engendrer des cycles selon des conditions à préciser ${ }^{25}$. Si les secteurs agricoles sont marqués par des oscillations importantes des prix, les secteurs industriels ne connaissent en revanche que des oscillations très faibles, du fait de l'ajustement continu ou graduel de l'offre. Pour traiter les interdépendances entre ces secteurs, Lange suggère de prendre en compte l'existence de délais d'ajustements non plus intra- mais intersectoriels.

"La même idée d'un ajustement continu ou progressif peut s'appliquer à la théorie de l'équilibre général. Cela doit alors être fait avec un flux continu - respectivement graduel - de capital et de travail d'une branche de production vers une autre et, ce faisant, un ajustement continu - respectivement graduel - de l'offre, qui atteint l'équilibre économique. La représentation mathématique de ce processus serait en tout cas très compliquée" (Lange [1935a], p. 363).

Les interdépendances entre les secteurs se traduisent par une mobilité progressive des facteurs de production d'un secteur à l'autre. La "force de travail" et les "capitaux" se déplacent progressivement en fonction des taux de rémunération proposés dans chaque secteur (ibid.). Les effets de reports d'un marché à un autre s'appréhendent ainsi à travers deux composantes : d'une part, leur durée, ou les délais nécessaires à la mobilisation des facteurs de production d'un marché à un autre et, d'autre part, le degré

25 La tentative n'est pas sans rappeler celle de Tinbergen [1930]. Ce dernier laisse en effet entrevoir la possibilité d'un traitement dynamique de l'ensemble de l'économie grâce à l'utilisation de formalismes mathématiques analogues au diagramme du cobweb. Voir Lendjel [1998], pp. 179-180. 
d'interdépendance entre ces deux marchés, selon le critère élaboré en $1932^{26}$. Ainsi, l'existence de délais d'ajustements inter-sectoriels est l'une des caractéristiques importantes du tâtonnement conçu comme cadre d'articulation des processus d'ajustement sectoriel. Dans cette perspective, l'inertie et le degré variable d'interdépendance entre les secteurs sont des facteurs de stabilité du processus général d'ajustement de l'offre et de la demande 27 .

Le tâtonnement, comme cadre, comporte une seconde caractéristique qui se profile derrière un élément favorisant la stabilité de l'équilibre général aux yeux de Lange. En effet, Lange considère que les secteurs n'ont pas tous le même poids dans l'économie. Dans cette perspective, la stabilité globale de l'économie dépend de la stabilité des secteurs les plus importants de cette économie. Cette idée, qui apparaît en 1935, sera reprise dans l'article de 1936-7. Ainsi, dans

"la grande majorité de la production économique [i.e. dans les secteurs industriels], l'ajustement de l'offre se fait continûment ou du moins progressivement" (Lange [1935a], p. 364)

Même si certains secteurs, comme l'agriculture, peuvent être caractérisés par un diagramme du cobweb de type oscillatoire, l'équilibre général sera pourtant stable, puisque

"le type marshallien d'adaptation de l'offre semble être dominant" (Lange [1936-7], p. 60, n. 1)

La convergence ou non du tâtonnement vers l'équilibre général dépendra pour partie du poids respectif des secteurs stables et des secteurs instables ${ }^{28}$. L'articulation des différents processus d'ajustement sectoriel dans le cadre du tâtonnement dépend donc également de l'importance relative des secteurs.

26 Notons qu'il faut attendre Valeur et Capital, de Hicks, pour que les effets de reports soient pleinement intégrés dans la théorie de l'équilibre général.

27 La réflexion de Lange sur cette question se déploiera pleinement en 1944. En 1941, Samuelson fait implicitement l'hypothèse que les vitesses d'ajustement des marchés sont identiques et constantes, de manière à retrouver les conditions de stabilité de Hicks. En 1944, Lange reprend cette formalisation du tâtonnement et met en lumière le rôle que peuvent jouer les vitesses d'ajustement dans l'évolution dynamique des marchés ([1944], pp. 95-9). Il montre que les conditions de Hicks sont compatibles avec des vitesses d'ajustement différentes des marchés. Notons qu'il existe des facteurs de stabilisation de l'équilibre pour Lange, comme les effets positif de la monnaie sur l'ajustement des variables (ibid., p. 12) ou le fait de supposer que certains biens ont des prix constants sur la période (ibid., p. 103, 106).

28 Lange mentionne un dernier facteur - typiquement marshallien - qui peut influencer la stabilité de l'équilibre. Il s'agit des anticipations réalisées par les entrepreneurs. En substance, Lange considère que l'entrepreneur ne peut pas manquer le point d'équilibre, en raison de la pente de la courbe de demande et du caractère progressif des adaptations de l'offre. En ce sens, le rôle des anticipations devient secondaire pour la question de la stabilité de l'équilibre (Lange [1935a], pp. 364-5). Le modèle développé par Lange est bien "marshallien" puisque, comme le dit R. Dos Santos Ferreira "Marshall ne suppose jamais la durée
Le portrait ainsi brossé par Lange du tâtonnement ressemble donc de moins en moins à celui de Walras. Par touches progressives, une représentation marshallienne du tâtonnement semble prendre forme. Le tâtonnement apparait comme un cadre qui coordonne des processus d'ajustement différents selon les secteurs. Ce cadre prend en compte des effets de masse, des processus d'ajustement de l'offre différents selon les secteurs, des vitesses d'ajustements différentes selon les secteurs, des délais variables d'ajustement intersectoriel et des degrés variables d'interdépendance entre les secteurs. Nous avons ainsi retrouvé un certain nombre de caractéristiques qui portent la marque distinctive de l'approche marshallienne selon De Vroey : "entrelacement de marchés séparés", rôle du temps "réel" dans la formation des grandeurs économiques, approche hiérarchisée de l'équilibre général, distinction entre équilibre de court et de long terme. Il ne manque plus que la composante organisationnelle qui figure au centre de l'article de 1936-7.

\section{Une lecture marshallienne du tâtonnement dans "On the Economic Theory of Socialism"}

Lorsque Lange s'insère dans le débat particulièrement vif sur la possibilité d'effectuer un calcul rationnel en économie socialiste, sa réflexion sur le tâtonnement est déjà assez avancée. Elle ne fait donc que se poursuivre dans "On the Economic Theory of Socialism" (1936-1937). Pourtant, la perspective a changé. Face aux critiques de Mises et de Hayek, Lange poursuit en effet deux objectifs précis qui ne sont plus simplement théoriques mais également organisationnels : d'une part, démontrer qu'il est possible d'obtenir un système de prix cohérent et "objectif" dans une économie socialiste et, d'autre part, montrer qu'une allocation rationnelle des ressources est "pratiquement" réalisable à partir de ce système de prix. Ce faisant, en mobilisant une seule procédure - la "procédure par essai et erreur" ou tâtonnement - pour répondre à ces deux objectifs, Lange lui confère apparemment une ambivalence qui se cristallise autour du statut de l'erreur. C'est ce que nous allons voir après avoir d'abord examiné les principales caractéristiques de cette procédure, pour montrer que cette ambivalence peut être levée avec une lecture marshallienne du tâtonnement.

\section{1. les caractéristiques formelles de la procédure par essai et erreur}

Les économies concurrentielles et socialistes se caractérisent avant tout par des organisations différentes de l'allocation des ressources. Les institutions, les dispositifs, les règles, les comportements et les procédures, en somme, l'organisation même des deux systèmes économiques est différente. Pourtant, affirme Lange, il existe une procédure que l'on peut appliquer à ces deux économies. La démonstration de Lange s'établit en trois temps. Dans un premier temps, il décrit le rôle et les caractéristiques de la procédure par "essai et erreur" dans une économie concurrentielle. Dans un deuxième temps, il montre que l'on peut

abolie au sein d'une période, même la plus courte. Le processus de production n'est jamais traité comme instantané; il en résulte notamment - et c'est le point qui nous concerne ici - que les producteurs prennent leurs décisions sur la base d'anticipations" (Dos Santos Ferreira [1989], p. 414). 
transposer cette procédure dans une économie socialiste, à condition d'organiser un système de règles cohérentes visant à obtenir des agents économiques un comportement similaire à celui adopté dans une économie concurrentielle. Le tâtonnement acquiert ainsi des caractéristiques organisationnelles distinctes selon les systèmes économiques. En cela, son approche du tâtonnement s'avère typiquement marshallienne. Dans un troisième temps, il trace les contours d'une procédure "formelle" que l'on peut appliquer à n'importe quel type d'économie. Cette procédure apparait alors comme un mode parmi d'autres d'allocation des ressources. Les deux premières étapes étant largement connues ${ }^{29}$, concentrons-nous sur la troisième, que Lange trace en pointillé.

Les caractéristiques "formelles" du tâtonnement s'obtiennent, selon Lange, en comparant l'usage de cette procédure en économie de marché et en économie socialiste. L'adjectif formel désigne, comme on l'a vu dans la première section, la dimension abstraite d'un phénomène par opposition à sa dimension "institutionnelle" ou "concrète" (Lange [1935b], p. 197).

Au niveau formel, les principes d'allocation des ressources sont identiques :

"[1]es principes formels sont les mêmes, mais la distribution réelle peut être complètement différente" (Lange [1936-7], p. 123).

Au niveau institutionnel, les caractéristiques du tâtonnement diffèrent selon les systèmes économiques. Pour Lange, ces "principes formels" de la distribution des ressources peuvent être dégagés par l'usage de l'analogie. En comparant les systèmes, on peut établir une analogie à un niveau très abstrait, une analogie "purement formelle" (ibid.). En termes mathématiques, cette analogie formelle revient à établir un isomorphisme, c'est-à-dire une correspondance biunivoque entre deux structures apparemment différentes. Cette forme d'analogie permet de dégager des principes qui sont identiques au niveau "formel" alors même qu'ils sont différents au niveau "réel".

La mise en correspondance analogique des principes comporte une conséquence importante pour la théorie socialiste, pour Lange

"[1]'analogie formelle [...] entre les principes de distribution des ressources dans un régime socialiste et ceux d'un régime concurrentiel d'entreprise privée, permet d'appliquer au premier régime la technique scientifique de la théorie de l'équilibre économique utilisée pour le second" (Lange [1936-7], p. 127).

Une fois cette analogie formelle constituée, on peut transférer la "technique scientifique de la théorie de l'équilibre" d'un système à l'autre ${ }^{30}$. En mathématique, un isomorphisme permet en effet de transférer les

29 Pour une analyse de ces deux premières étapes, voir Lendjel [1998], pp. 236-254.

30 Cette opinion semble partagée par de nombreux auteurs de la période, notamment en ce qui concerne l'application des catégories des théories de l'équilibre aux économies socialistes. Pour eux, ces concepts sont en effet, comme le dit Knight, des concepts universels : "les principes du marginalisme sont les principes logiques, mathématiques, et ainsi universels, de l'économie; [...] Et cette condition générale et fondamentale ne serait en rien changée par le remplacement de la concurrence des individus sur les propriétés et théorèmes d'une structure à une autre. Ainsi, en appliquant la technique des théories de l'équilibre à l'économie socialiste ${ }^{31}$, on transfère également les théorèmes qu'elles ont établis. Par exemple, la propriété d'optimalité de l'équilibre en concurrence parfaite se retrouve dans une économie socialiste ayant mis en place une procédure de distribution des ressources analogue à celle du tâtonnement ${ }^{32}$. On peut alors recenser les caractéristiques formelles du tâtonnement à partir de l'étude des différents cas où Lange l'utilise.

- Le tâtonnement est avant tout considéré par Lange comme une "procédure par essai et erreur" (ibid., pp. 57, 67-68). C'est ce caractère procédural qui rend possible son application à des économies différentes et qui permet, simultanément, d'en lister les caractéristiques formelles.

En tant que procédure sociale, le tâtonnement mobilise un certain nombre d'acteurs : des ménages, des entrepreneurs et une "instance" qui impulse la procédure par des essais, et la corrige lorsqu'elle constate des erreurs. A proprement parler, la concurrence n'est pas une "instance" et Lange n'évoque jamais la présence d'un "commissaire priseur" qui l'incarnerait ${ }^{33}$. Pourtant, elle est formellement identique aux actions du Centre.

L'objet testé - à l'aide d'essais - dans cette procédure est un système de prix conçus comme des "indices de choix alternatifs", c'est-à-dire en tant qu'indicateurs de la rareté relative des biens à usages alternatifs (ibid., p. 54) ${ }^{34}$. Ces prix, testés d'abord "au hasard" (ibid., p. 57) par l'instance centrale, mesurent la rareté relative des biens, afin d'en permettre la répartition optimale entre tous les acteurs. A

marchés par le collectivisme, ou en modifiant la forme de l'organisation économique dans tout autre sens" (Knight [1936], p. 256).

31 Lange affirme même que les caractéristiques d'une économie socialiste se prêtent particulièrement bien aux techniques développées par les théoriciens de l'équilibre comme Walras ou Marshall (Lange [1936-7], p. 127). Ainsi, "[1]e système capitaliste réel est beaucoup mieux décrit par les analyses de Mrs. Robinson et du Professeur Chamberlin" (ibid.). Lange rejoint ici Dickinson : "[1]es magnifiques systèmes de l'équilibre économique décrits par Böhm-Bawerk, Wieser, Marshall et Cassel, ne sont pas des descriptions de la société telle qu'elle est, mais des visions prophétiques de l'économie socialiste du futur" (Dickinson [1933], p. 247).

32 Roberts est très critique vis-à-vis de l'application du tâtonnement à une économie socialiste. Ainsi, "[l]a simulation du marché est ainsi confondue avec une planification centrale. Il n'est évidemment pas venu à l'esprit de nombreux auteurs qu'une simulation d'un système doit refléter la structure de ce système. Une simulation du marché reflète la structure du marché et les relations d'échanges de la production des marchandises" (Roberts [1971], p. 573, note 9).

33 Certains auteurs sont persuadés qu'il existe, dans la représentation que propose Lange d'un marché concurrentiel, un commissaire priseur. Voir ainsi Vaughn [1980], p. 541, ou Cave et Hare [1981], p. 4.

34 Littéralement, "terms on which alternatives are offered". La terminologie vient de P. H. Wicksteed [1910], p. 217. La traduction est empruntée à Brus [1961], p. 49. 
travers les prix, l'instance teste donc la cohérence et l'optimalité d'une certaine répartition des ressources rares.

Pour être testé, il faut au préalable communiquer le système de prix à tous les acteurs. Ce point ne soulève pas a priori de difficultés majeures, sauf que cette tâche doit être assumée par une instance spécifiée. Une fois l'information communiquée aux acteurs, leurs réactions doivent obéir à quatre principes préétablis.

1/ Leurs réactions ne doivent dépendre que de l'information prix qu'ils ont reçue. Pour cela, deux conditions sont nécessaires : d'une part, les prix doivent comporter une fonction paramétrique, selon laquelle les prix s'imposent aux agents comme des paramètres dans leurs calculs (Lange [1936-7], pp. 57, 63), d'autre part, le revenu des agents doit être réductible à des prix (la "condition sociale (C)" décrivant le mode de formation des revenus) (ibid., pp. 57, 61).

2/ La réaction des agents doit être conforme à l'objectif recherché. S'il s'agit d'obtenir la répartition la plus cohérente et la plus satisfaisante des ressources disponibles, il faut doter les agents de certaines règles de décisions basées sur l'utilisation la plus rationnelle possible des ressources. Si chaque agent utilise au mieux les ressources qu'il gère, alors la répartition sociale de ces ressources peut devenir optimale. C'est précisément la fonction des "conditions subjectives (A)" formulées par Lange (ibid., pp. 57, $62)$.

3/ Les règles suivies par les agents doivent être compatibles entre elles. Il ne sert à rien, par exemple, d'organiser la liberté du choix de l'occupation (ibid., p. 62) si l'on impose pas en parallèle au responsable de la production la règle d'égalité du prix et de la productivité marginale du facteur (Lange [1936-7], p. 124)

4/ Les réactions des agents doivent être manifestes, que ce soit par la mise en ouvre de la production et des échanges, ou par de simples engagements conditionnels, dont la réalisation dépend de la validité du système de prix proposé par le Centre (ibid., p. 64).

Les réactions quantitatives ne sont prises en considération qu'au terme de la période d'ajustement des agents, après avoir eux-mêmes procédés à une série d'essais et d'erreurs pour trouver leur situation optimale. Lorsque ces réactions se sont manifestées, elles sont confrontées sous la forme d'une rencontre entre une offre et une demande. Ces réactions sont donc synthétisées par l'agrégation des propositions quantitatives individuelles (ibid., p. 67). Ce moment synthétique est effectué par l'instance centrale (concurrence ou Centre) de manière à évaluer la validité de l'essai initial ${ }^{35}$.

L'erreur se manifeste lorsqu'il se produit un écart entre l'offre et la demande effectifs de biens et services. En cas d'écart, l'instance centrale réagit en faisant varier les prix en fonction du signe de la demande nette (la "condition objective (B)") (ibid., pp. 57-60, ). Elle teste ainsi un nouveau système de prix qu'elle soumet aux agents économiques. $\mathrm{Si}$, à ce nouveau système de prix, les demandes nettes s'annulent, la procédure s'arrête puisque le système de prix proposé correspond à une répartition optimale

35 Pour certains auteurs, comme Roberts ([1971], p. 568), le Centre est un organisme "redondant," puisqu'il assume des tâches qui peuvent être spontanément assumées par le marché, sans recours à une administration. C'est, nous semble-t-il, nier ce que dévoile Lange, à savoir que le tâtonnement a logiquement besoin d'une instance centralisatrice pour fonctionner. des ressources disponibles. Autrement, l'instance centrale propose un nouveau système de prix aux agents en suivant la même règle d'ajustement. La règle de la demande nette incarne donc pratiquement à elle seule le déroulement de la procédure, dans la succession de ses étapes ainsi que dans son arrêt.

La procédure de tâtonnement a des propriétés qui sont transférables d'une économie à une autre, du fait de l'existence d'une "analogie formelle" - ou isomorphisme - entre ces économies. Le tâtonnement comporte principalement deux propriétés.

1/ Il répartit les ressources disponibles de manière cohérente en conciliant la structure de la demande et celle de l'offre. Lorsque ces structures émanent de choix individuels, le tâtonnement rend ces choix de consommation, d'occupation et de production compatibles entre eux.

2/ Il assigne une valeur sociale, objective, à tout élément de la richesse sociale, qu'elle soit marchande ou non. C'est ainsi une procédure d'objectivation des prix, où l'objectivité s'acquiert par le ralliement progressif de tous les agents à leur valeur. Au fur et à mesure des progrès de la procédure, de plus en plus d'agents deviennent satisfaits des prix proposés. Les prix seront totalement objectifs quand ils seront adoptés par tous, assurant la compatibilité de tous leurs choix.

3/ Il définit des valeurs uniques et stables à tout élément de la richesse sociale. Le système de prix qu'il propose au terme de son déroulement est un système unique d'équilibre, d'où aucune force ne cherche à l'en éloigner.

Par delà les différences organisationnelles propres à chaque système économique, le tâtonnement apparaît ainsi comme une procédure formelle que l'on peut appliquer à des systèmes économiques distincts. Cela étant, les caractéristiques de cette procédure laissent planer un doute sur le caractère effectif ou non des erreurs consécutives aux essais de l'instance centrale. Examinons plus avant ce point.

\subsection{Le statut de l'erreur dans le tâtonnement de Lange}

Lange semble hésiter entre deux interprétations du tâtonnement. Certains éléments, comme la prise en compte des délais de production, tendent à accréditer lidée que la procédure par essai et erreur tolère l'existence d'une production et d'échanges hors équilibre. L'erreur nécessaire au déroulement du tâtonnement de Lange ne peut ainsi s'évaluer "sur le papier", selon l'expression de Barone ([1908], § 56, p. 295), mais doit pouvoir s'observer empiriquement. D'autres éléments au contraire accréditent l'idée que le tâtonnement ne tolère aucun échange avant l'équilibre. Il serait donc aussi atemporel qu'il l'est dans la conception walrassienne. L'enjeu de cette alternative se situe dans le fait de savoir si le tâtonnement - en tant que procédure "pratique" - peut retrouver la solution calculée "théoriquement" à partir d'un système d'équation ou non. En accentuant la dimension organisationnelle du tâtonnement, Lange prend le risque de 
supprimer l'identité établie par Walras entre solution "théorique" et solution "pratique" des systèmes d'équations 36

Examinons les arguments en présence.

- $\quad$ Du premier côté, on trouve deux éléments plaidant dans le sens d'une dimension temporelle du tâtonnement avec mise en œuvre des échanges et de la production pendant la durée du processus d'ajustement.

1/ Le premier élément provient de l'interprétation par Lange de la distinction établie par Walras entre la détermination "théorique" de l'équilibre et sa détermination "pratique". Contrairement à Walras, Lange doit relever le défi hayekien et proposer une procédure concrète d'allocation rationnelle des ressources, notamment en économie socialiste. Il s'agit de contourner l'obstacle d'une résolution pratique des équations qui deviendrait obsolète avant même d'être résolue.

"Cela nécessiterait l'établissement de millions d'équations à partir de millions de données statistiques basées sur de nombreux autres millions de calculs individuels.

Durant la période nécessaire à la résolution des équations, l'information sur laquelle elles seraient basées serait devenue obsolète et elles devraient de nouveau être calculées" (Lange [1936-7], p. 56).

Il doit donc montrer "comment le problème est réellement résolu par essai et erreur" (ibid., p. 59).

En réponse à Hayek et Robbins, Lange soutient que le Centre ne résout aucune équation, puisque ce sont les agents qui s'en chargent.

"Les consommateurs les "résolvent" en dépensant leur revenu de manière à obtenir le maximum total d'utilité; et les gestionnaires des unités de production les "résolvent" en trouvant la combinaison de facteurs et l'échelle de production qui minimise le coût moyen" (ibid., p. 67).

Il s'agit du même mode de résolution que celui régnant dans le système "actuel", lorsque Hayek résout une équation lorsqu'il achète un journal (ibid.). De ce fait, la procédure par essai et erreur ne peut se faire de manière fictive puisque personne ne connaît sa fonction de demande ou d'offre.

"Pour établir les prix qui servent de paramètres aux personnes pour "résoudre les équations", aucune mathématique n'est non plus nécessaire. Nul besoin de connaître des fonctions d'offre et de demande. Les prix adéquats sont simplement trouvés en examinant les quantités demandées et les quantités offertes et en augmentant le prix d'une marchandise ou d'un service lorsqu'il se produit un excès de demande sur

36 Nous avons montré ailleurs (Lendjel [1999]) que Walras tentait de démontrer l'identité entre ces deux solutions en s'appuyant sur la méthode itérative de Lagrange. Cette méthode assure, sous certaines conditions, que le tâtonnement parvient "en pratique" à la même solution que celle calculée par le théoricien. l'offre et en le baissant dans le cas inverse, jusqu'à ce que, par essai et erreur, le prix soit atteint lorsque la demande et l'offre sont égales" (ibid., p. 67).

En l'absence d'une connaissance de ces fonctions, les agents ne peuvent que réagir par une demande ou une offre face à un système de prix qui leur est proposé. Ainsi, l'erreur, et notamment celle des unités de production, est nécessairement réelle, puisque, sans cela, les agents ne pourraient déterminer leurs "conditions subjectives" d'équilibre.

2/ Lange soutient que toute erreur dans la répartition des ressources engendre de graves conséquences en économie socialiste tout comme en économie de concurrence (ibid., p. 66). L'existence de goulots d'étranglement devient rapidement dirimante pour la poursuite du processus productif, puisqu'en cas de pénurie d'un facteur, la production s'arrête si l'unité productive n'arrive pas à trouver de facteurs substituables. Ainsi :

"Toute erreur faite par le Ministère de la Planification Centrale en fixant les prix se manifesterait d'une manière très objective : par une pénurie ou un surplus physique de la quantité de la marchandise ou de la ressource en question, et devrait être corrigée pour conserver un fonctionnement régulier de la production" (Lange [19367], p. 64).

L'erreur ne serait donc pas virtuelle, mais "objective" puisque se manifestant "physiquement" par un surplus ou une pénurie. De surplus en déficit, d'erreur en erreur, la procédure conduit à trouver les solutions d'équilibres.

Ces deux éléments tendent donc à montrer que la procédure ne s'appuierait pas sur de simples propositions d'offre et d'achat, susceptibles d'être révisées à tout moment, mais au contraire sur des transactions effectives entre agents.

- $\quad$ A l'inverse, certains éléments plaident en faveur du caractère fictif des essais et des erreurs dans la procédure ${ }^{37}$. Deux éléments peuvent être retenus en ce sens.

1/ Si Lange distingue, à la suite de Pareto et de Barone, la détermination théorique de l'équilibre de sa détermination effective ou "réelle", il doit logiquement supposer que les deux solutions - théorique et effective - sont identiques. La méthode par essai et erreur doit permettre de retrouver les solutions des équations de l'équilibre. Ainsi,

"Barone a déjà souligné le fait que les équations de l'équilibre économique doivent être également résolues dans une société socialiste par essai et erreur" (Lange [19367], p. 56).

${ }^{37}$ Hayek ([1940] p. 131), Schumpeter ([1947], pp. 234-235), et plus tard Cave et Hare ([1981] p. 101) partagent tous la même interprétation de l'article de Lange sur la nature fictive des tentatives effectuées par le Centre. 
Or, comme le fait remarquer Hayek, la détermination théorique de l'équilibre s'effectue dans un cadre où toutes les données du problème sont constantes (Hayek [1940], p. 131) ${ }^{38}$. Il ne peut donc se produire d'échanges avant l'équilibre si l'on veut obtenir une solution effective identique à la solution théorique, sauf à supposer que les échanges effectifs ne modifient pas les données du problème.

2/ L'aspect séquentiel du tâtonnement plaide également pour la virtualité des essais. En effet, Lange sépare nettement les différentes étapes de la procédure, supposant implicitement que le passage à l'étape suivante dépend de l'accomplissement de l'étape précédente. Une fois les prix fixés, ils ne peuvent plus varier tant que la demande et l'offre ne se sont pas manifestés (Lange [1936-7], p. 63). L'intervention de l'instance centrale qui centralise ces offres et demande et qui met en œuvre la règle de la demande nette s'inscrit dans la même perspective. Ces étapes sont agencées selon un temps logique qui semble incompatible avec la simultanéité d'opérations différentes. Là encore, les essais ne peuvent donc qu'être fictifs, puisqu'ils nécessitent le recours à la clause de suspension des échanges hors de l'équilibre.

Il existe donc une ambivalence dans l'interprétation que l'on peut donner du tâtonnement chez Lange. D'un côté, le tâtonnement comporte une dimension temporelle, caractérisée par la mise en œuvre irréversible de la production et des échanges avant l'obtention de l'équilibre. De l'autre, le tâtonnement ne peut qu'être logiquement atemporel, empêchant toute forme de réalisation des engagements contractés par les agents pendant toute la durée du processus. Peut-on lever cette ambivalence?

\subsection{Lever l'ambivalence par une lecture marshallienne du tâtonnement ?}

En fait, on s'aperçoit que les indices qui véhiculent l'ambivalence mentionnée dépendent principalement de nos lunettes walrassiennes. En effet, ces lunettes induisent la nécessité de recourir à une clause de suspension des échanges avant l'équilibre pour que la solution "pratique" - par tâtonnement - soit identique à la solution "théorique" - fournie par les systèmes d'équations -. Or, comme l'a signalé récemment De Vroey, l'approche marshallienne n'a pas besoin de cette clause pour assurer l'identité des deux solutions.

L'approche marshallienne met plutôt en avant trois hypothèses qui fonctionnent conjointement. En premier lieu vient l'hypothèse d'absence d'effets de report dans le temps ("no-carry-over"), comme l'a souligné Kaldor ([1934], p. 127). Cette hypothèse se justifie lorsque les biens sont rapidement périssables. Dans cette configuration, les agents ne prennent en considération dans leurs plans que les données de la période en cours et agissent en conséquence. Mais cette condition est insuffisante en elle-même pour assurer l'identité des solutions théoriques et pratiques, pour reprendre la terminologie de Walras. En effet, les échanges à faux prix induisent des effets de revenus susceptibles de conduire les agents à réviser leurs

38 En effet, le principal reproche que Hayek adressera, en 1940, à la solution de Lange, est précisément celui de son caractère "statique", sans correspondance avec le "monde réel" (Hayek [1940], p. 131). C'est également ce que lui reproche Lerner [1937]. Selon ce dernier, l'analyse du socialisme s'avère biaisée en raison de "la nature statique de l'équilibre concurrentiel" (Lerner [1937], p. 253). Voir également Peter Murrell [1983], p. 97 et Gabriel Temkin [1989], pp. 202-206. plans pendant le processus d'ajustement. Pour neutraliser ces effets, il faut introduire une deuxième hypothèse : la constance de l'utilité marginale de la monnaie (idem., p. 128). Sur un marché isolé, la quantité de monnaie détenue par les agents représente en effet leur revenu disponible pour les échanges (ibid., Lange [1936-7], p. 57) ${ }^{39}$

"En conséquence, la possibilité d'effets revenus est écartée. Dans ce cas, l'échange à faux prix peut se produire. Puisqu'il ne s'accompagne pas d'une dépendance du chemin [path-dependency], le marché se clôturera avec la même quantité échangée que dans la situation d'information parfaite avec le dernier échange se produisant au prix 'véritable d'équilibre' de Marshall" (De Vroey [1999b], p. 171).

Selon Kaldor, cette hypothèse se justifie par le fait que la marchandise échangée sur le marché considéré ne représente qu'une petite fraction du revenu des agents, rendant ainsi négligeable les effets revenus induits par des échanges à faux prix (Kaldor [1934], p. 128, n. 3). Enfin, une troisième hypothèse s'avère également nécessaire :

"l'approche marshallienne est étayée par l'hypothèse de constance des données. [...]

Les données économiques sont supposées rester constantes durant le laps de temp du processus d'ajustement afin d'assurer le succès de l'ajustement vers l'équilibre (que ce soit de courte ou de longue période)" (ibid., p. 172).

Les "données" auxquelles fait référence Kaldor sont les caractéristiques des fonctions d'offre et de demande ou, autrement dit, les préférences des agents et les techniques productives. Dans cette perspective, les échanges et productions en déséquilibre deviennent possibles durant le processus d'ajustement, tout en conservant l'identité des solutions théorique et pratique ${ }^{40}$. Dès lors, une lecture marshallienne de la procédure par essai et erreur de Lange - ou tâtonnement, selon son terme - devient possible.

Peut-on la retrouver en 1936-7 ? Tout d'abord, les trois hypothèses figurent au moins implicitement dans cet article. La deuxième, relative à la constance de l'utilité marginale de la monnaie, apparaît à au moins deux reprises. Lors de l'analyse de la concurrence, Lange liste les conditions "subjectives" de l'équilibre. Parmi celles-ci,

"[1]es consommateurs maximisent l'utilité totale qu'ils dérivent de leur revenu en le dépensant de telle sorte que l'utilité marginale du montant procurable pour une unité de revenu (exprimé en monnaie) est égal pour toutes les marchandises" (Lange [1936-7], p. 57).

Cette phrase indique implicitement que l'unité de monnaie est l'étalon qui permet à chaque consommateur de comparer le montant des biens entre eux. Cette unité étant logiquement constante, elle équivaut donc à poser l'hypothèse de la constance de l'utilité marginale de la monnaie. Plus loin, lors de l'étude des

39 Cette hypothèse interprétative est largement connue. Voir, par exemple, Hicks [1939], pp. 114-116 Diatkine [1982], p. 70, ou Lallement [1985], pp. 162-8.

40 Pour une analyse de la dimension temporelle des ajustements et l'absence de clause de suspension des échanges chez Marshall, voir également Diatkine [1982] et Dos Santos Ferreira [1989], pp. 420 et suivantes. 
avantages de l'économie socialiste, Lange fait l'hypothèse que l'on peut comparer les utilités individuelles entre elles, afin de pouvoir calculer le maximum de bien-être dans une économie planifiée (ibid., p. 123, n. 1). Et, pour être encore plus explicite, pose comme nécessaire l'hypothèse selon laquelle "l'utilité marginale du revenu est la même pour tous les consommateurs" (ibid., p. 124). Là encore, cette hypothèse n'est que la transposée socialiste de l'hypothèse de la constance de l'utilité marginale de la monnaie ${ }^{41}$. Les deux autres hypothèses marshalliennes ne figurent pas explicitement dans l'article de 1936-7, mais s'avèrent implicitement nécessaire à l'argumentation de Lange. En effet, ces hypothèses interviennent dès lors qu'il a recours au diagramme du cobweb et au processus d'ajustement marshallien (Lange [1936-7], p. 60, n. 1). Ces deux représentations requièrent toutes deux l'hypothèse de "constance des données" et de "no-carryover" pour que le processus d'ajustement puisse arriver à son terme.

A ce versant hypothétique s'ajoute le soin qu'apporte Lange au versant organisationnel dans ses réflexions sur le fonctionnement possible d'une économie socialiste. Ce versant - largement connu contribue à doter le tâtonnement de caractéristiques clairement non-walrassiennes.

Enfin, au-delà de ces hypothèses et des caractéristiques organisationnelles, Lange se réfère explicitement à ses travaux antérieurs dans une note (Lange [1936-7], p. 60, n. 1) pour préciser les conditions de stabilité de l'équilibre sur un marché. Lange soutient en effet qu'une "modification de la quantité offerte requiert généralement une période de temps" (Lange [1936-7], p. 60 note 1). Il montre, en s'appuyant sur ses travaux de 1935, que l'offre s'adapte par paliers successifs à une modification initiale de la demande, et parvient au bout d'un certain temps à la situation d'équilibre. Les délais de production se répercutent donc sur le temps mis par la procédure pour parvenir à l'équilibre. Des échanges sont donc nécessaires à son déroulement, avant même d'avoir atteint l'équilibre. On retrouve donc bien ces éléments marshalliens en 1936-7.

Nous avions alors envisagé la possibilité selon laquelle le tâtonnement représenterait, pour Lange, un cadre analytique dans lequel s'inscrirait de multiples processus d'ajustement différents selon les secteurs. Cette possibilité s'affirme plus nettement encore en 1936-7 lorsque Lange recense trois des avantages d'une économie socialiste. 1/ Comme le Centre n'a pas besoin de résoudre une seule équation, l'économie socialiste ne perd pas plus de temps à calculer la solution d'équilibre que le marché concurrentiel. La vitesse de réaction des agents n'a aucune raison de différer d'un système à l'autre, puisque le type d'information et son mode de traitement s'avère identique. 2/ Davantage, du fait des connaissances macro-économiques du Centre, le temps d'ajustement du système entier doit être plus court dans un système socialiste. En effet, par la prééminence de sa position, le Centre est en mesure de réduire le nombre d'essais nécessaires au système pour parvenir à la solution d'équilibre (Lange [1936-7], p.67). De surcroît,

"ce processus d'essai et erreur se déroule, bien sûr, sur la base de prix historiquement donnés. Des ajustements relativement petits de ces prix se feraient constamment, et il

${ }^{41}$ Lange déploie d'ailleurs un argumentaire assez tortueux pour justifier cette hypothèse (Lange [1936-7], p. 125). En l'espèce, s'il n'y a aucune raison a priori pour que l'utilité marginale du revenu soit identique d'un individu à l'autre, Lange soutient que les sensibilités individuelles se distribuent autour d'une loi normale. De là se déduit une courbe moyenne permettant les calculs d'optimisation du bien-être collectif. ne serait pas nécessaire de construire un système de prix entièrement nouveau"

(Lange [1936-7], p. 66)

3/ Certaines branches peuvent être affectées par le caractère cyclique de leur production, et décrire ainsi des fluctuations conformes au diagramme du cobweb. C'est le cas, par exemple, de certaines industries agricoles. Le Centre peut alors réduire l'amplitude des cycles oscillatoires en anticipant l'effet des prix sur le volume de la production mise en œuvre (Lange \& Taylor [1938], p. 90), et peut même éviter que l'instabilité d'une branche ne se répercute sur l'ensemble de l'économie (Lange [1936-7], p. 126).

Derrière ces trois avantages, le tâtonnement apparaît donc bien comme un cadre qui articule des processus d'ajustements de l'offre différents selon les secteurs considérés, selon des vitesses d'ajustement intersectoriel variables. Relevons ici que l'impact du poids relatif d'un secteur sur la stabilité de l'économie ne joue plus dans le cadre d'une économie socialiste. Le tâtonnement en tant que cadre d'analyse dépend ainsi lui-même des caractéristiques organisationnelles de l'économie.

A travers cette lecture marshallienne du tâtonnement, Lange pense avoir ainsi résolu la tension entre la dimension mathématique du tâtonnement, qui justifie la convergence du processus marchand vers l'équilibre, et sa dimension organisationnelle, qui justifie sa dimension "pratique" 42 . Il s'agit là du principal avantage du syncrétisme de Lange consistant à vouloir résoudre des problèmes économiques en brassant des concepts issus de cadres conceptuels distincts.

\section{Conclusion}

Pour conclure, nous formulerons deux brèves remarques.

En replaçant l'article de 1936-7 dans la démarche intellectuelle de Lange, nous avons montré la prégnance des théories de Marshall dans sa réflexion sur le tâtonnement. Une interprétation marshallienne du tâtonnement semble ainsi se dégager. Le tâtonnement apparaît alors comme un cadre, caractérisé avant tout par la fonction paramétrique des prix conjuguée avec la règle de la demande nette. Ce cadre articule des processus d'ajustements de l'offre différents selon les marchés, où les marchés sont caractérisés par leur importance relative et leur isolement relatif par rapport aux autres marchés. En somme, les contours de ce cadre dépendent des caractéristiques organisationnelles des économies et secteurs considérés. La stabilité de l'équilibre général ne se pose donc plus, pour Lange, en termes généraux, mais dépend de ces caractéristiques organisationnelles.

42 De nombreuses objections ont été soulevées à l'encontre du modèle de Lange, comme l'applicabilité des règles de décision à la marge (Roberts ([1971], p. 571) ou l'absence d'incitations pour les responsables de la production. Pour une appréciation critique du modèle de Lange, voir Bergson [1948], pp. 434-439, [1967], pp. 659-660. Mais d'après J. Steindl : "[c]e sont des détails. La grande idée fut de proposer un mécanisme administratif rationnel fait par l'homme, reposant sur le principe du signal de retour [feed-back principle], un contrôleur, pour assurer le fonctionnement régulier et efficient d'une économie socialiste complexe et hautement développée : une véritable piece d'ingénierie sociale" (Steindl [1964], p. 554). 
Il reste toutefois un problème d'exégèse à résoudre. Si Lange semble développer une lecture marshallienne du tâtonnement dans ses premiers écrits, sa lecture du tâtonnement s'inscrit résolument, dans Price flexibility and employment (1944), dans une perspective walrassienne. Comment expliquer ce basculement ? Un élément de réponse peut être avancé. En 1941, l'article de Samuelson va complètement modifier la manière de penser le tâtonnement. La fascination de Lange pour les mathématiques (Brus et Kowalik [1970], pp. 156), que l'on avait déjà relevé dans son article de 1932, ainsi que les nouvelles opportunités qu'il entrevoit l'ont probablement incité à changer son fusil d'épaule. En effet, Lange trouve dans cette nouvelle formalisation un moyen pour poursuivre sa réflexion sur le rôle des vitesses d'ajustement dans la stabilité de l'équilibre général. Il relève ainsi, en 1944, que les résultats de Samuelson reposent sur l'hypothèse de constance des vitesses d'ajustement et montre que l'on peut conserver les conditions de Hicks avec des vitesses d'ajustement différentes selon les marchés. On relèvera également que la seule modification qu'introduit Samuelson lors de la reprise de son article de 1941 dans ses Foundations en 1947 concerne .... les vitesses d'ajustement ${ }^{43}$. On relèvera enfin que le tâtonnement est revenu sur le devant de la scène sous une forme marshallienne, du fait du syncrétisme de Lange, alors que la postérité n'en a retenu, à la suite de Samuelson, que la version walrassienne.

\section{Bibliographie}

BARONE Enrico [1908], "Il Ministro della Produzione nello Stato Collettivista", Giornale degli Economisti sept.-oct. 1908, réédité in F. von Hayek [1935].

BergSON Abram [1948], "Socialist Economics," in H. S. Ellis (ed.) A Survey of Contemporary Economics, vol. 1, pp. 412-448, publié par The American Economic Association, Richard D. Irwin Inc, Homewood, Illinois, 11 ème édition, 1966.

Bergson Abram [1967], "Market Socialism Revisited", The Journal of Political Economy, 75 (5), October 1967, pp. 655-673.

Brus Wlodzimierz [1961], Problèmes généraux du fonctionnement de l'économie socialiste, traduit du polonais par B.P. Leblanc, S. Nowocien, Anna Posner, série "économie et socialisme," vol. 10, Paris, Maspero, 1970.

BRUS Wlodzimierz, KowaliK Tadeusz [1970], "L'économie et le socialisme selon Oskar Lange", Cahiers de l'I.S.E.A., Economies et Sociétés, tome IV, nº 1, janvier 1970, pp. 147-184

CAVE Martin, Hare Paul [1981], Alternative Approaches to Economic Planning, MacMillan, London, 1981.

De VROEY Michel [1999a] "Transforming Walras into a Marshallian Economist : A Critical Review of Donald Walker's Walras's Market Models", Journal of the History of Economic Thought, 21 (4), pp. 413-435.

\footnotetext{
43 Voir Lendjel [1998], pp. 330-337.
}

De Vroey Michel [1999b] "Equilibrium and Disequilibrium in Economic Theory : A Confrontation of the Classical, Marshallian and Walras-Hicksian Conceptions", Economics and Philosophy, 15 (1999), pp. 161-185.

DE VROEY Michel [1999c] "The Marshallian Market and the Walrasian Market Economy : Two Incompatible Bedfellows", Scottish Journal of Political Economy, 46, pp. 319-38.

DiATKINE Daniel [1982], "La formation du prix sur «le marché du blé» selon A. Marshall," Cahiers $d^{\prime E}$ Economie Politique, n 7 , PUF, Paris, 1982, pp. 67-92

DickINSON H. D. [1933], "Price Formation in a Socialist community," The Economic Journal, vol. XLIII, june 1933, pp. 237-250.

DoNZELLI Franco [1993] "The Influence of the Socialist Calculation Debate on Hayek's View of General Equilibrium Theory", Revue Européenne des sciences sociales, 31 (96), pp. 47-83.

DOS SANTOS FerReira Rodolphe [1989], "Equilibre marshallien et équilibre walrasien", Recherches Economiques de Louvain, 1989, 55 (4), pp. 399-424.

EllotT, John E. [1976], "Marx and Contemporary Models of Socialist Economy", History of Political Economy, vol. 8, n² 2, Summer 1976, pp. 151-184.

FRIEDMAN M. [1949], "The Marshallian Demand Curve", Journal of Political Economy, 57, pp. 463-95.

GISLAIN Jean-Jacques, STEINER Philippe [1995], La sociologie économique : 1890-1920, Paris, PUFSociologies, 1995.

GRANGER G.G. [1955], Méthodologie Economique, Paris, PUF, 1955

HAYEK Friedrich August (ed.) [1935], Collectivist Economic Planning, London, Routledge, 1935, traduit en français in L'économie dirigée en régime collectiviste, éd. Politique Economiques et Sociologiques, Paris 1939.

HAYEK Friedrich August [1940], "Socialist Calculation : The Competitive 'Solution'", Economica, vol. vII, $\mathrm{n}^{\circ}$ 26, May 1940, pp. 125-149.

HiCKS J. R. [1939], Valeur et Capital, Paris, Dunod, 1981.

KALDOR Nicholas [1934], "A classificatory Note on the Determinateness of Equilibrium", The Review of Economic Studies, fev. 1934, 1 (2), pp 122-136.

KNIGHT Frank [1936], "The Place of Marginal Economics in a Collectivist System", The American Economic Review, supplément, 26 (1), Mars 1936, pp. 255-266.

KowALIK, Tadeusz [1987a] "Lange, Oskar Ryszard (1904-1965)", The New Palgrave, édité par J. Eatwell, M. Milgate et P. Newman, Macmillan Press, Londres, pp. 123-129.

KowaLIK, Tadeusz [1987b] "Lange-Lerner mechanism", The New Palgrave, édité par J. Eatwell, M. Milgate et P. Newman, Macmillan Press, Londres, pp. 129-131.

LALLEMENT Jérôme [1985], "Alfred Marshall : l'utilité mesurée comme une grandeur de la physique", Economia, Economies et Sociétés, Série P.E., n 3, mars 1985, pp. 147-171.

LANGE Oskar [1932], "Die allgemeine Interdependenz der Wirtschaftsprognosen und die Isolierungsmethode", Zeitschrift für Nationalökonomie, 4 (1), pp. 52-78.

LANGE Oskar [1935a], "Formen der Angebotsanpassung und wirtschaftliches Gleichgewicht," Zeitschrift für Nationalökonomie, pp. 358-365. 
LANGE Oskar [1935b], "Marxian Economics and Modern Economic Theory", The Review of Economic Studies, vol. II, $\mathrm{n}^{\circ}$ 3, June 1935, pp. 189-201.

LANGE Oskar [1936-1937], "On the Economic Theory of Socialism", The Review of Economic Studies, vol. IV, pp. 53-71 (part I) et pp. 123-142 (part II).

LANGE Oskar [1944], Price Flexibility and Employment, Bloomington, Indiana Principia Press, 1944.

LANGE Oskar, TAYLOR Fred M. [1938], On the Economic Theory of Socialism, New York, Augustus M. Kelley, 1970.

LavoIE Don [1985], Rivalry and Central Planning. The Socialist Calculation Debate Reconsidered, Cambridge, Cambridge University Press, 1985.

LENDJEL Emeric [1998], Les métamorphoses du tâtonnement : une histoire de ses formalisations de Walras à Samuelson, Thèse de Doctorat, Université de Paris I Panthéon-Sorbonne.

LENDJEL Emeric [1999], "Tâtonnement walrassien et marchandage parétien : une approche comparative", in $\mathrm{P}$. Bridel et $\mathrm{E}$ Tatti (eds.) L'équilibre général entre économie et sociologie, Revue Européenne des Sciences Sociales, 37 (116), pp. 295-314.

LEONTIEF Wassily [1934], "Verzögerte Angebotsanpassug und partielles Gleichgewicht", Zeitschrift für Nationalökonomie, Band V, Heft 5, 1934, p. 670-676, traduit et réédité dans Essais d'Economiques, Paris, Calmann-Lévy, 1974, pp. 303-314.

LERNER Abba P. [1937], "Statics and dynamics in socialist economics", The Economic Journal, June 1937, vol. XLVII, ${ }^{\circ} 186$, pp. 253-270.

MENARD Claude [1990] L'économie des organisations, Paris, La Découverte-Repère, 1990.

Mises Ludwig von [1920], "Die Wirtschaftsrechnung im Sozialistichen Gemeinwesen," Die Archiv für Sozialwissenschaften, Vol. 47, 1920, réédité in Hayek (ed.) [1935], pp. 93-132.

MuRRELL Peter [1983], "Did the Theory of Market Socialism Answer the Challenge of Ludwig Von Mises? A Reinterpretation of the Socialist Controversy", History of Political Economy, 15 (1), Spring 1983, pp. 92-105.

PALERMO G. [1998] "The socialist planning debate : a historical and analytical reconsideration", History of Economic Ideas, 6 (3), pp. 115-150.

REBEYROL Antoine [1999] La pensée économique de Walras, Paris, Dunod, 1999.

RICCI Umberto [1930], "Die «synthetische Ökonomie» von Henry Ludwell Moore", Zeitschrift für Nationalökonomie, I bild, n ${ }^{\circ}$, Heft II, 1930, p. 649-679.

ROBERTS Paul Craig [1971], "Oskar Lange's Theory of Socialist Planning", The Journal of Political Economy, 79 (3), May-June 1971, pp. 562-577.

Rosenstein-RodAn P. N. [1929], "Das Zeitmoment in der mathematischen Theorie des wirtschaftlichen Gleichgewichtes", Zeitschrift für Nationalökonomie, Bild 1, Heft 1, 1929, pp. 129-142.

RothBARD Murray N. [1976], "Ludwig Von Mises and Economic Calculation Under Socialism", in Laurence S. Moss (ed.), The Economics of Ludwig Von Mises : toward a critical reappraisal, Studies in Economic Theory, Kansas City, Sheed and Ward inc., 1976

SCHUMPETER Joseph A. [1947], Capitalisme, socialisme et démocratie, Paris, Payot, $2^{2}$ ème éd. française, 1990.
StEINDL Josef [1964], "Servo-Mechanisms and Controllers in Economic Theory and Policy", On Political Economy and Econometrics : Essays in Honour of Oskar LANGE, Pergamon Press, Polish Scientific Publishers, 1964, pp. 545-554.

TAYLOR Fred M. [1929], "The Guidance of Production in a Socialist State," The American Economic Review, 19 (1), Mars 1929, pp. 1-8, discours présidentiel présenté au 41 ème congrès annuel de l'American Economic Association, Chicago, Illinois, le 27 decembre 1928, réédité in Lange et Taylor [1938].

TemKIN Gabriel [1989] "On Economic Reforms in Socialist Countries : the Debate on Economic Calculation Under Socialism Revisited", Communist Economies, 1 (1), pp. 31-59, réédité in A. Nove et I. D. Thatcher (eds.) [1994] Markets and Socialism, Aldershot, Edward Elgar, 1994, pp. 196-224.

TINBERGEN Jan [1930], "Bestimmung und Deutung von Angebotskurven : Ein Beispiel", Zeitschrift für Nationalökonomie, I bild, ${ }^{\circ}{ }^{5}$, Heft II, 1930, pp. 669-679.

VAUGHN Karen I. [1980], "Economic Calculation under Socialism : the Austrian Contribution", Economic Inquiry, 18 (4), October 1980, pp. 535-545.

WALKER Donald A. [1997] Advances in General Equilibrium Theory, Cheltenham, Edward Elgar, 1997.

Weintraub E. Roy [1991], Stabilizing Dynamics; Constructing economic Knowledge, Cambridge, Cambridge University press, 1991.

WiCKSTEED Philip H. [1910], The common sense of Political Economy, Routledge \& Kegan Paul, London, 1967. 